\title{
Mesophase study of pure and doped cyanobiphenyl liquid crystals with salen type systems
}

Amal Bendahou ${ }^{1,2}$, Zoulikha Khouba ${ }^{1,2}$, Tayeb Benabdallah ${ }^{2}$, Ulrich Maschke ${ }^{1 *}$

${ }^{1}$ Unité Matériaux et Transformations - UMET (UMR CNRS No8207), Bâtiment C6, Université Lille 1 - Sciences et Technologies, 59655 Villeneuve d'Ascq Cedex, France

${ }^{2}$ Laboratoire de Chimie et d'Electrochimie des Complexes Métalliques (LCECM), Université des Sciences et de la Technologie d'Oran Mohamed Boudiaf, USTO-MB, BP 1505,

El-M’Naouer, 31000 Oran, Algeria

e-mail : ulrich.maschke@univ-lille1.fr 


\begin{abstract}
In the current study, two salenSchiff base ligands derived from the condensation of ethylendiamine on salicylaldehyde and 5-chlorosalicylaldehyde, namely, N,N'-bis( salicylidene)ethylene-diamine(L1) and N,N'-bis(5-chlorosalicylidene)ethylene-diamine (L2)as well as two of their iron(III) and nickel(II) complexes were prepared, and then used as doping agents of two thermotropic liquid crystals of cyanobiphenyle type, namely, 4-cyano4'-n-pentyl (5CB) and 4'-n-octyl-4-cyano-biphenyl (8CB). The study of the mesophase of pure and doped liquid crystals was carried out by UV-visible spectroscopy equipped with a heating compartment for precise temperature control, differential scanning calorimetry and polarized optical microscopy. The characteristic transition temperatures, nematic-isotropic, $\mathrm{T}_{\mathrm{NI}}$, and/or smectic-nematic, $\mathrm{T}_{\mathrm{SN}}$, of liquid crystals based systems were measured and then compared to those of the literature concerning pure $5 \mathrm{CB}$ and $8 \mathrm{CB}$. Optical microscopy has shown the existence of Schlieren and focal conic textures of the nematic and smectic states respectively, both of pure and doped liquid crystals. The homogeneity of the obtained guesthost systems was proven by the linear evolution of their transition temperatures as function of the concentration in solute, with correlation factors near to unity.
\end{abstract}

Keywords: Schiff base, guest-host, Liquid Crystal, phase transition,nematic state, transition temperature, smectic state, texture of mesogenic systems. 


\section{Introduction}

Liquid crystals (LCs) are an important class of materials, known for their anisotropic character, conferring them various physical properties. They are applied in many fields of display and visualization technology, such as liquid crystal displays (LCDs) and polymer dispersed liquid crystals (PDLCs) [1-3]. Some LCs has useful semiconducting properties, suggesting many applications in the field of molecular electronics. They have the required characteristics for use as active layers in photovoltaic devices [4, 5]. LCs also possess have also several applications in data storage field, according to new holographic techniques $[6,7]$. A large amount of work is focused on changing and improving the optical properties of LCs by chemical means such as synthesis, and physical way such as doping. The chemical method gives rise to a wide range of new LC molecules, having varied structures, containing different aliphatic chains, aromatic rings and different functional groups, and characterized by new nematic and/or smectic textures [8-10].

Recently, dispersion of solutes in LCs seems to be the most suitable method for obtaining new mesogenic composite materials, known as guest-host systems, having new optical and physico-chemical properties. Among the many doping molecules, which have been subject of guest-host systems, one finds various dyes such as rhodamines and coumarin $[11,12]$, nanoparticules [13] and carbone nanotubes [14-16]. Due to their absorbance in the visible region, the dye doped LCs are the subject of several studies, in particular concerning intermolecular interactions of the dipole-dipole type, hydrogen bonding, aggregates and $\pi-\pi$ stacking. These interactions are expressed in terms of absorption bands undergoing blue or red shifts, modification in their shape and/or intensity, during the passage from the isotropic phase to the nematic one, and vice versa, as well as the changing in their order parameter values $[17,18]$. Among the many systems cited in the literature, and employed as guests for mesogenic hosts, Schiff bases are not largely explored as doping molecules except in few reportsappearing in 
the literature [19-21]. Unlike dyes, aromatic Schiff base systems are easy to prepare, and feature high thermal and photonic stabilities, allowing them to be used as innovative molecules in the guest-host study.Schiff baseThe structures of these molecules present a variety of functional groups, aromatic parts and aliphatic chains, conducting to different interactions with LCs.

Moreover, it is known that Schiff bases play an important role in chemistry, especially in liquid-liquid extraction [22, 23], coordination in solid and liquid states [24-26], catalysis and corrosion inhibition [27-30], antibacterial and antifungal activities, etc.[31-34].

The motivation of the study comes from the fact that the salen Schiff base derivatives as well as their metal complexes are known for their high thermal and photonic stability, unlike some systems used as dopants (dyes by way of example). This makes it possible to envisage their use as innovative molecules in the guest-host study requiring a long exposure to the solar rays (solar panels for example) or under high temperatures. Such systems can therefore be used for the preparation of eutectic LCs whose nematic phase is characterized by a wide temperature range. This is all the more justified as the LCs doped with these solutes exhibit no phase separation in the chosen concentration range.

In this paper, spectral and thermalbehavior of two Schiff bases, namely,N,N'bis(salicylidene)ethylene-diamine(L1), N,N'-bis(5-chlorosalicylidene)ethylene-diamine (L2) (Figure 1) and two of their complexes, L1-iron(III) and L2-nickel(II), were studied in different isotropic and anisotropic media. The present work was undertaken in order to observe the effect of doping agents on the mesophase of pure 5CB and $8 \mathrm{CB}$ LCs. All the results obtained by UV-visible spectroscopy, differential scanning calorimetry and polarised optical microscopy were compared with those previously reported in the literature, concerning the same pure LCs. 


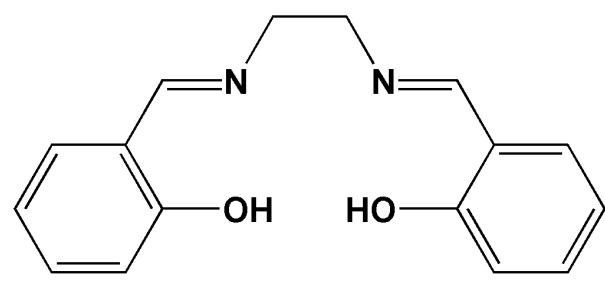

L1

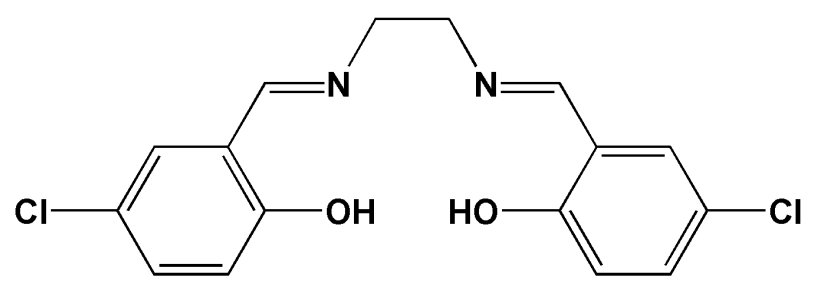

$\mathbf{L 2}$
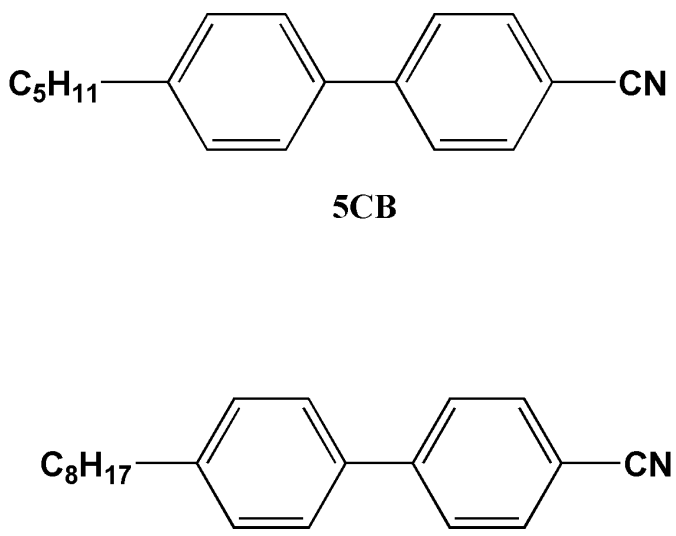

8CB

Figure 1 : Chemical structures of the Schiff bases (L1 and L2) and the LCs (5CB and 8CB).

\section{Experimental}

\subsection{Materials and methods}

All the isotropic solvents, with high purity (spectroscopic grade) were purchased from Sigma Aldrich. The pure nematic liquid crystals (5CB and $8 \mathrm{CB}$ ) used in our experiments as anisotropic media are from Synthon Chemicals Germany.

The Schiff bases N,N'-bis(salicylidene)ethylene-diamine(L1), N,N'-bis(5chlorosalicylidene)ethylene-diamine (L2) and their iron(III) and nickel(II) complexes were 
synthesized and purified according to the general method described in the literature [35-37], and were used as guest materials. All systems were analyzed by infrared spectroscopy, gravimetric thermal analysis and nuclear magnetic resonance $\left({ }^{1} \mathrm{H}\right.$ and $\left.{ }^{13} \mathrm{C}\right)$.

It should be noted that only L1 Schiff base (98\% purity) and its nickel complex (96\% purity) used in this study are commercially available (Alfa Aesar and Sigma Aldrich). The same ligands and some of their iron(III), cupper(II) and cobalt(III) complexes have also been the subject of several works in the literature $[26,38]$. The results of their infrared, $\mathrm{H}^{1}-\mathrm{NMR}$ and $\mathrm{C}^{13}$-NMR are almost identical to those presented in this paper, which confirms the purity of our products $[35,36]$.

\subsection{Preparation of the guest-host systems}

After synthesis and characterization of the ligands and their complexes, the guest-host systems were prepared by dispersing a small quantity of each of these solutes in the LCs. The solute concentration range was situated-is between 0.05 and $1.4 \mathrm{wt} \%$. The systems are then placed under mechanical stirring in a temperature-controlled room (about $25^{\circ} \mathrm{C}$ ) for about 48 hours to ensure their homogenization.

\subsection{Techniques}

\subsubsection{Infrared Spectroscopy}

The guest systems were analyzed by Fourier-transform infrared spectroscopy (FTIR spectroscopy) using a Bruker ALPHA spectrometer equipped with a Platinum ATR single reflection diamond attenuated total reflectance module (Perkin-Elmer, FTIR spectrometer Frontier). The number of accumulated scans was 16.

\subsubsection{Nuclear Magnetic Resonance}


${ }^{1} \mathrm{H}-\mathrm{NMR}$ and ${ }^{13} \mathrm{C}-\mathrm{NMR}$ spectra were acquired on a FT-NMR(800MHZ) Bruker instrument. Deuterated Dimethylsulfoxide (with $0.06 \%$ Tetramethylsilan) was used as NMR solvent and was obtained from Eurisotop (Saclay, France) and used as received.

\subsubsection{Thermal analysis}

The thermal study of the solutes were performed using a Perkin Elmer thermogravimetric Analyzer Pyris1TGA from 20 to $900^{\circ} \mathrm{C}$, at a heating rate of $10^{\circ} \mathrm{C} / \mathrm{min}$, under nitrogen atmosphere.

\subsubsection{UV-visible spectroscopy}

The electronic spectra of all systems have been recorded by using a Varian Cary 100 UVvisible double-beam spectrophotometer, equipped with a heating accessory for precise temperature control $\left( \pm 0.1^{\circ} \mathrm{C}\right)$. The measurements were made as a function of temperature in Hellma quartz cells (100-QS) of 1 and 10mm thickness, for mesogenic and isotropic solvents respectively.

The UV-visible spectra for measuring the transition temperatures were obtained in the first heating mode, from 25 to $50^{\circ} \mathrm{C}$, using the steps of 0,5 to $0,1^{\circ} \mathrm{C}$ and an appropriate hold time (1min) for each scan, to obtain a thermodynamic stable state, in the range of 200 to $800 \mathrm{~nm}$. This ramp is so spread out over time that the system is permanently in a thermodynamic equilibrium, so that cooling or heating mode give exactly the same results and can be compared to those of DSC and POM.

The thermal study was undertaken by varying the temperature from 25 to $50^{\circ} \mathrm{C}$, with steps of 0.5 to $0.1^{\circ} \mathrm{C}$ using appropriate hold time $(1 \mathrm{~min})$ to obtain a thermodynamic stable state, in the range of 200 to $800 \mathrm{~nm}$. 


\subsubsection{Differential Scanning Calorimetry (DSC)}

DSC measurements were performed on a Perkin-Elmer PYRIS Diamond DSC apparatus. Temperature and heat flow scales were calibrated with high purity indium standards. Samples of about $6 \mathrm{mg}$ were analyzed under nitrogen gas flow at a heating rate of $10^{\circ} \mathrm{C} / \mathrm{min}$, from -72 to $60^{\circ} \mathrm{C}$, held at the latter for $2 \mathrm{~min}$, cooled back to $-72^{\circ} \mathrm{C}$, followed by an isothermal scan for $2 \mathrm{~min}$. The same steps were repeated during a second and a third heating/cooling cycle. The thermograms discussed here correspond to the second heating cycle.

\subsubsection{Polarized Optical Microscopy (POM)}

The polarized optical microscopy (POM) is a common technique that gives information on the transition temperatures and morphology of materials such as LCs and PDLC systems.

The POM measurements were realized on an Olympus BX-41, equipped with a heating/cooling stage Linkam LTS 350, a Linkam TMS 94 temperature control unit and a digital camera that can record images with high resolution, conjugated with a computer.

A droplet of the sample is placed on a microscopic slide of about 4-6 $4 \mathrm{~m}$ and heated from room temperature $\left(25^{\circ} \mathrm{C}\right)$ to a temperature of $50^{\circ} \mathrm{C}$, so that the mixture became isotropic, then another slide was put on the first, and all is maintained at $50^{\circ} \mathrm{C}$ for about $5 \mathrm{~min}$, before cooling the sample down until the room temperature, at using a rate of $0.1^{\circ} \mathrm{C} / \mathrm{min}$.

During the first cooling mode eperation, the transition temperatures of the systems are observed and the pictures of their textures are taken at the different states and temperatures.

\section{Results and discussion}

\subsection{Characterization of the Schiff bases and their metal complexes}

The Schiff bases L1 and L2, obtained as light yellow crystals, with 92 and $81 \%$ yield respectively, were re-crystallized in ethanol. Their melting points are 126 and $157^{\circ} \mathrm{C}$ 
respectively. Their ${ }^{1} \mathrm{H}-\mathrm{NMR}$ spectra (figures $\mathrm{S} 1$ and $\mathrm{S} 2$ ), recorded in $\mathrm{d}_{6}$-DMSO solvent exhibit a massif within the range $6.86-7.55 \mathrm{ppm}$ which is assigned to aromatic protons. The azomethine protons $(-\mathrm{CH}=\mathrm{N}-)$ appeared as a singlet at $8.6 \mathrm{ppm}$. The signals situated at 13.37$13.44 \mathrm{ppm}$ are characteristic of phenolic protons. The aliphatic protons appeared as singlet at 3.94ppm (s, 4H, $-\mathrm{CH}_{2}-\mathrm{CH}_{2}-$ ). The ${ }^{13} \mathrm{C}-\mathrm{NMR}$ spectra of the Schiff bases (figures S3 and S4) show the signals of aliphatic carbons (-C-C-) situated at 58.46-58.72ppm and the aromatic ones at 116.43-132.32ppm. The azomethine and phenolic carbons appear respectively at 166.89 and $160.54 \mathrm{ppm}$ for L1 and at 165.74 and $159.53 \mathrm{ppm}$ for L2. IR spectra of the free ligands (figures S5 and S6) revealed the absorption bands of the azomethine groups (-CH=N-) at $1637-1632 \mathrm{~cm}^{-1}$ as shown by Aranha and coll. [36]. These frequencies are shifted to the lower values in the spectra of their corresponding complexes which appeared at 1629 and $1623 \mathrm{~cm}^{-1}$ for L1-Fe (figure S7) and L2-Ni (figure S8) respectively. Such behaviour has often been encountered in the literature $[38,39]$ Streh a result and suggests the coordination of the azomethine groups to the metal center. The thermal analysis (TGA) of the solutes, realized between 20 and $900^{\circ} \mathrm{C}$, in inert atmosphere, show the degradation temperature values for L1, L2, L1-Fe and L2-Ni beyond 211, 222, 265 and $273^{\circ} \mathrm{C}$, respectively (figures S9 to S12). These values are much higher than the temperature range of our study $\left(25-50^{\circ} \mathrm{C}\right)$ and ensure the thermal stability of our systems during the heat treatment.

\subsection{Solvent effect on the absorption spectra of the systems in the visible region}

The electronic spectra of all solutes in ethanol, dimethylsulfoxide (DMSO) and acetonitrile, as isotropic solvents, and in $5 \mathrm{CB}$ and $8 \mathrm{CB}$ as LCs, were obtained in the isotropic state, at $42^{\circ} \mathrm{C}$ (figures 2 and 3). As shown in figure 2, the spectra of L1(figure 2a) and L2(figure 2b) present a single absorption band situated at $405-421 \mathrm{~nm}$, in ethanol and DMSO, reflecting a ketonic form of the two Schiff bases, in equilibrium with the enolic one, situated below $400 \mathrm{~nm}$, such 
has been observed for other Schiff bases[40]. This band is transformed into a shoulder in $5 \mathrm{CB}, 8 \mathrm{CB}$ and acetonitrile, which contain a cyanide group $(-\mathrm{C} \equiv \mathrm{N})$. This can be explained by the passage of the intra-molecular interaction between $\mathrm{OH}$ and imine groups, in the case of ethanol and DMSO, to the inter-molecular interaction between the cyanide and $\mathrm{OH}$ groups, in the case of the LCs and acetonitrile.

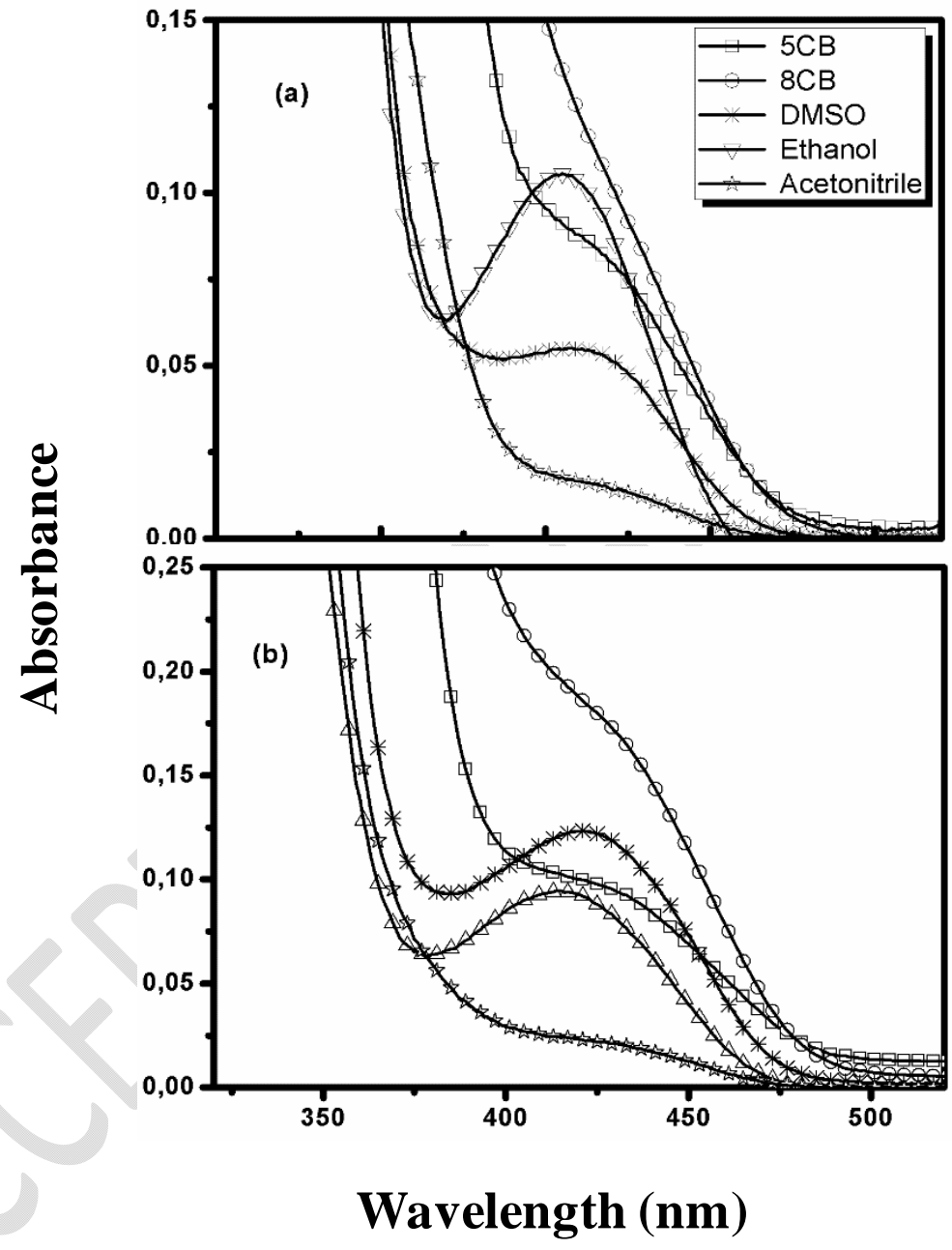

Figure 2 : Electronic spectra of (a) L1 and (b) L2 in different media, at $42^{\circ} \mathrm{C}$.

The absorption spectra of $\mathrm{L} 1-\mathrm{Fe}$ and $\mathrm{L} 2-\mathrm{Ni}$ complexes, recorded at $42^{\circ} \mathrm{C}$, in the same solvents, except 8CB (lack of solubility), are presented in figure 3 . 
The iron(III) and nickel(II) complexes are characterized by the d-d transitions which are interpreted in terms of absorption band in the visible region of the spectra, at wider wavelengths (Figure 3) than those of free ligands (Figure 2). On the other hand, these systems are strongly colored so that a very low concentration of solute makes it possible to observe very clearly the absorption band of the visible and to better visualize the texture of the LC by optical microscopy (Figure 8).

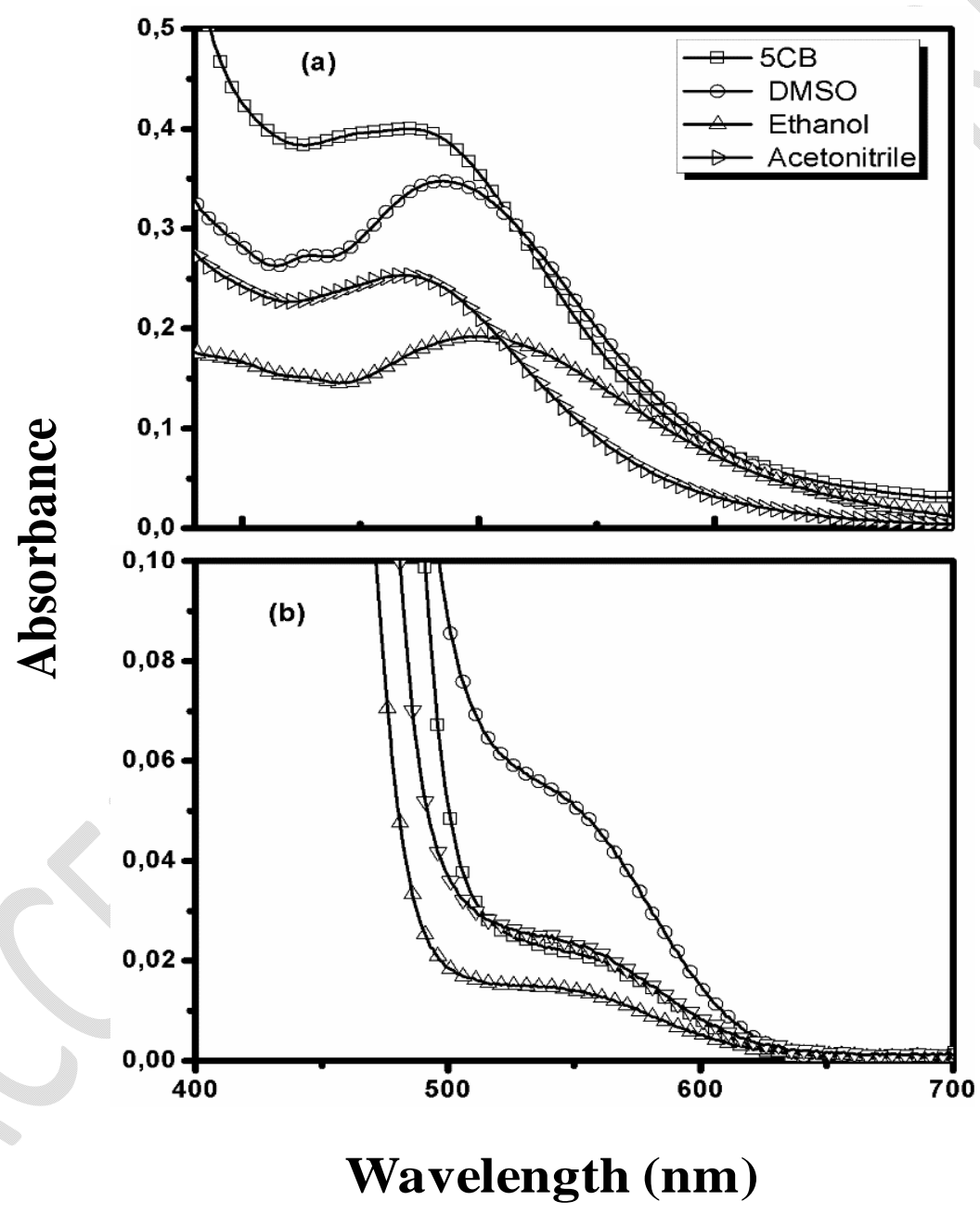

Figure 3 : Electronic spectra of (a) [L1-Fe] and (b) [L2-Ni] complexes in different media, at $42^{\circ} \mathrm{C}$. 
A single absorption band is obtained in each solvent and situated between 468 and 508nm, for L1-Fe complex, reflecting an octahedral geometry around the metal center. For L2-Ni complex, this band is transformed into a shoulder, in the same solvents, at higher frequency (536-550nm) and at 536nm in DMSO and ethanol for $\mathrm{L} 2 \mathrm{Ni}$ complex, reflecting a square planar geometry around $\mathrm{Ni}^{2+}$ ion. In $5 \mathrm{CB}$ and acetonitrile, the band is transformed into a shoulder at the same frequency, referring to the same type of geometry as in ethanol and DMSO. In this case From these results, we can then conclude that the LCs act as the usual isotropic solvents toward the complexes, without specific interactions.

\subsection{Investigation of the phase transition of pure and doped liquid crystals using different}

\section{techniques}

\subsubsection{UV-visible spectroscopy}

The study of the mesophase of pure and doped LCs, 5CB and 8CB, was first undertaken by UV-visible spectroscopy. This technique is still little exploited in the literature despite its simplicity and reliability [19]. The spectra were obtained in the range of the temperature between 25 to $50^{\circ} \mathrm{C}$ with step of $0.1^{\circ} \mathrm{C}$ around the phase transition. The concentration of the solutes varied in general from 0.05 to $1.4 \mathrm{wt}-\%$. The metal complexes do not have the same solubility in LCs as their corresponding ligands, so the concentration effect of the complexes could not be studied and compared to that of the Schiff bases.

The evolution of absorbance, measured at $600 \mathrm{~nm}$, as a function of temperature was plotted and presented in figures 4 and 5.

The plots show distinguishable levels characterizing the different phases of the LC based system. These latter appear at different absorbance values which vanish in the isotropic state. In general, the increase in Schiff base concentration gradually decreases the $\mathrm{T}_{\mathrm{NI}}$ and $\mathrm{T}_{\mathrm{SN}}$ values of the different systems with regards of the pure LC. Contrary to the ligands, the 
complexes L1-Fe and L2-Ni show a slight increase in the $\mathrm{T}_{\mathrm{NI}}$ value of $5 \mathrm{cb}$ for the low solute concentration $(0.05 \mathrm{wt}-\%)$, this inverse observed phenomenon requires a more detailed study in the future work.

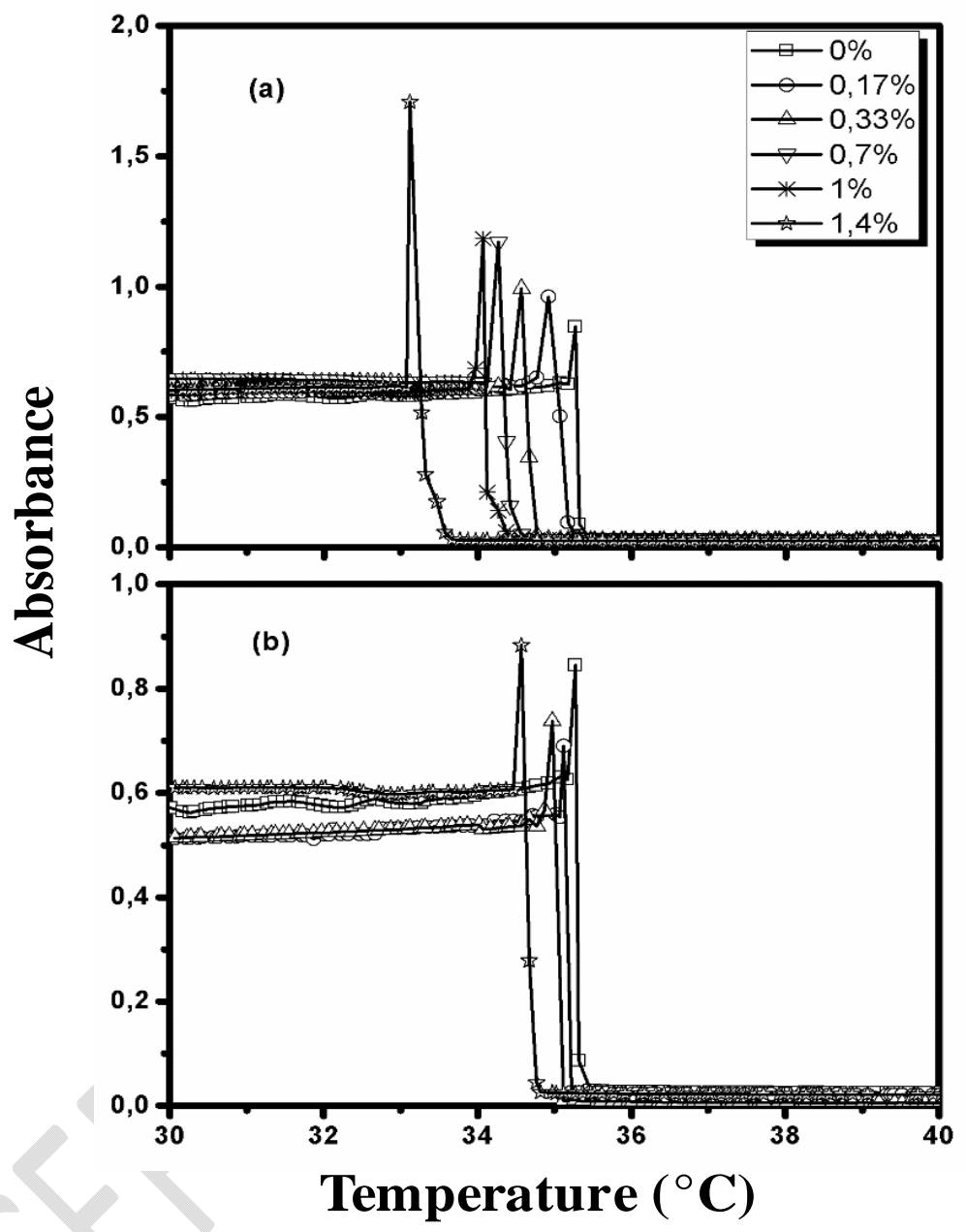

Figure 4 : Variation of the absorbance at $600 \mathrm{~nm}$ against temperature, of pure and doped 5CB by (a) L1 and (b) L2, at different concentrations. 


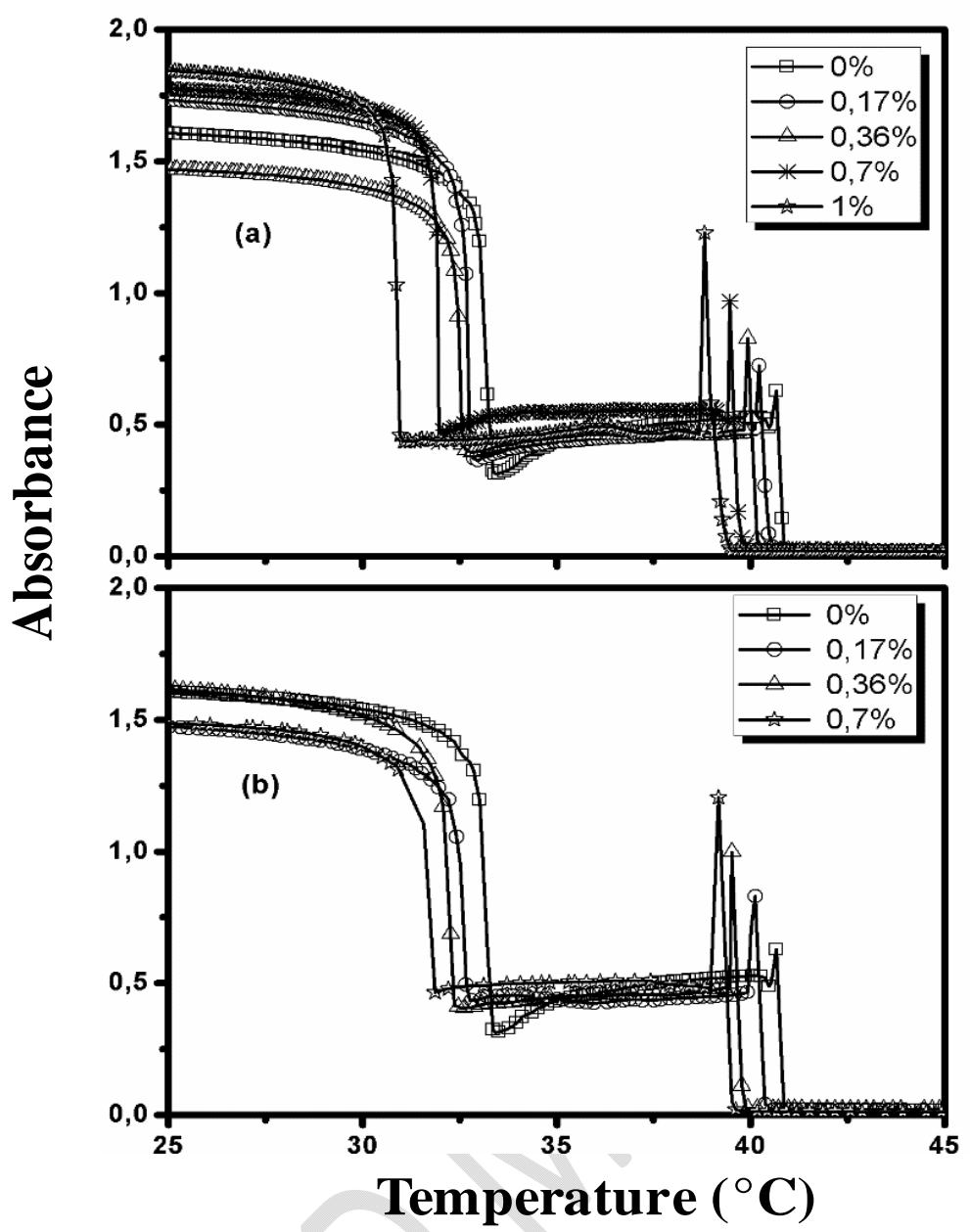

Figure 5 : Variation of the absorbance at $600 \mathrm{~nm}$ against temperature, of pure and doped $8 \mathrm{CB}$ by (a) L1 and (b) L2, at different concentrations.

Figure 6 presents the variation of $\mathrm{T}_{\mathrm{NI}}$ values as function of concentration in ef solute, for L1-5CB and L2-8CB systems as examples, and obtained from both UV-visible and DSC techniques for comparison. In general, this evolution proves to be linear, justifying the homogeneity of the LC systems, which is interpreted by the regular dispersion of the solute molecules in the LCs. The slight deviation from the linearity observed for DSC data in the case of L1-8CB (Figure 6a), can be explained by the influence of apparatus parameters used in each case. 
The characteristic temperatures of all systems are gathered in the table 1 tables 1 and 2 . The $T_{N I}$ and $T_{S N}$ values are calculated using the following formulas: $T_{N I}=1 / 2\left(T_{N}+T_{I}\right)$ and $T_{S N}=$ $1 / 2\left(\mathrm{~T}_{\mathrm{S}}+\mathrm{T}_{\mathrm{N} 0}\right)[19,41]$, where $\mathrm{T}_{\mathrm{S}}$ and $\mathrm{T}_{\mathrm{N}}$ represent the last points of the levels of the smectic and nematic phases, respectively. As for $\mathrm{T}_{\mathrm{N} 0}$ and $\mathrm{T}_{\mathrm{I}}$, they correspond respectively to the first points of the nematic and isotropic levels.

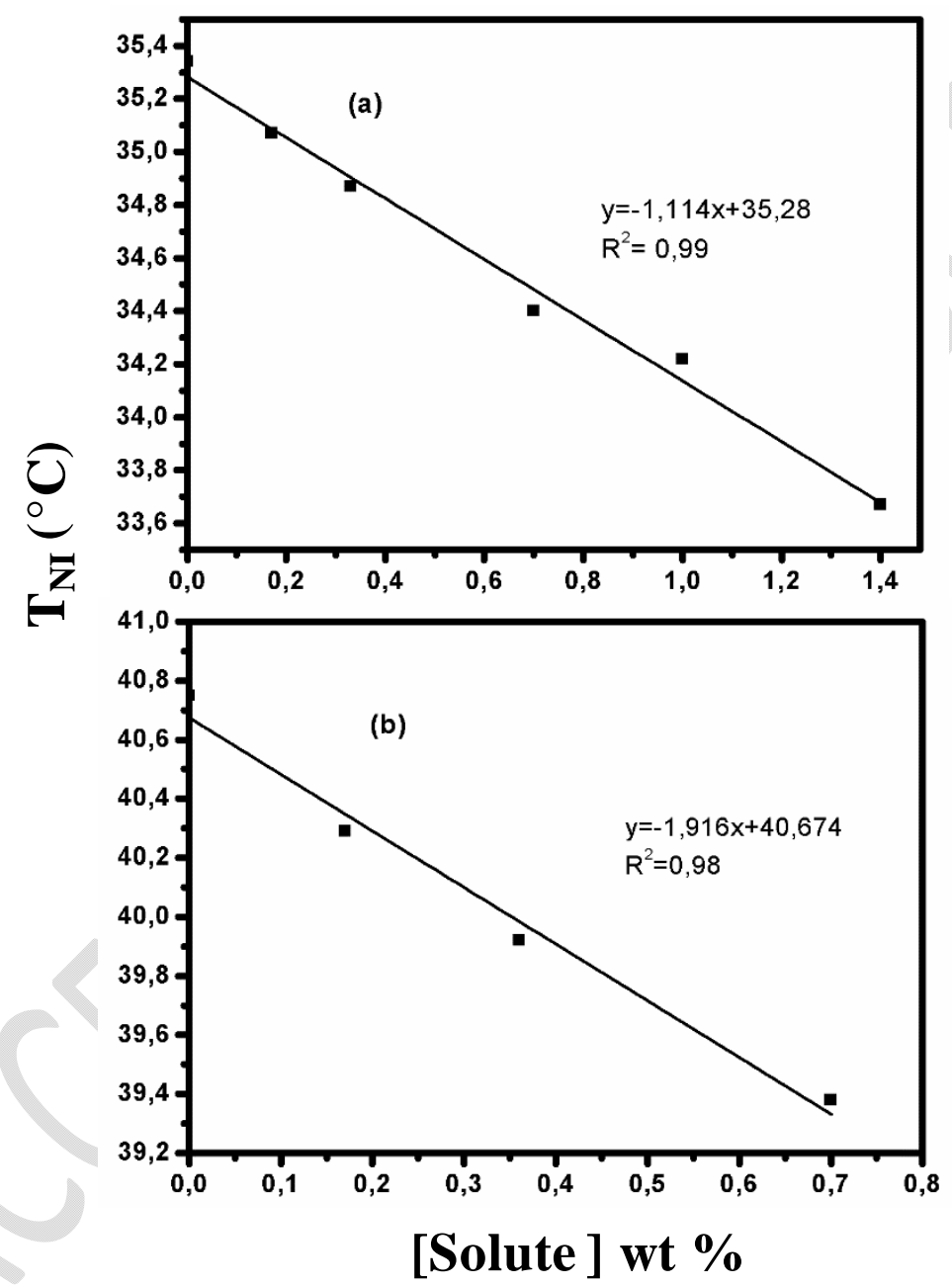

Figure 6 : Variation of the $\mathrm{T}_{\mathrm{NI}}$ of (a) [L1-5CB] and (b) [L2-8CB] depending on the solute concentration, established by UV-visible spectroscopy. 
Table 1: Characteristic temperatures of solute-5CB and solute- $8 \mathrm{CB}$ systems, obtained by UVvisible spectroscopy.

\begin{tabular}{|c|c|c|c|c|c|c|c|c|}
\hline Solute-5CB & Wt-\% & $\mathrm{T}_{\mathrm{NI}}$ & $\Delta \mathrm{T}_{\mathrm{NI}}$ & Solute-8CB & $\mathbf{W t - \%}$ & $\mathrm{T}_{\mathrm{SN}}$ & $\mathrm{T}_{\mathrm{NI}}$ & $\Delta \mathrm{T}_{\mathrm{NI}}$ \\
\hline Pure 5CB & 0,00 & 35,3 & - & Pure 8CB & 0,00 & 32,9 & 40,8 & - \\
\hline L1-5CB & 0,17 & 35,0 & 0,3 & L1-8CB & 0,17 & 32,4 & 40,4 & 0,4 \\
& 0,33 & 34,9 & 0,4 & & 0,36 & 32,0 & 40,0 & 0,8 \\
& 0,70 & 34,4 & 0,9 & & 0,70 & 31,5 & 39,7 & 1,1 \\
& 1,00 & 34,2 & 1,1 & & 1,00 & 30,6 & 39,1 & 1,7 \\
& 1,40 & 33,7 & 1,6 & & & & & \\
& & & & & & & & \\
\hline L2-5CB & 0,17 & 35,2 & 0,1 & L2-8CB & 0,17 & 32,2 & 40,3 & 0,5 \\
& 0,33 & 35,0 & 0,3 & & 0,36 & 31,9 & 39,7 & 1,1 \\
& 0,70 & 34,6 & 0,7 & & 0,70 & 31,3 & 39,4 & 1,4 \\
& & & & & - & - & - & - \\
\hline L1Fe-5CB & 0,05 & 35,6 & $-0,3$ & - & - & - & - & - \\
\hline L2Ni-5CB & 0,05 & 35,7 & $-0,4$ & - & & - & & \\
\hline
\end{tabular}

The peculiarity of the UV-visible technique consists in the possibility of observing the fluctuation phenomenon around the nematic-isotropic transition of all systems. This can be interpreted, from their UV-visible spectra, in terms of the abrupt increase in absorbance and its drop to near zero, just before the passage to the isotropic state, in a relatively low temperature range of about 0,1 or $0,2^{\circ} \mathrm{C}$. Such a phenomenon is at the origin of the discontinuous character of the N-I transition, both for the pure and doped LCs. in LCs in generat.

A fluctuation phenomenon was observed at the nematic-isotropic transition of all systems andinterpreted, in theirUV visible spectra, by the abrupt increase of the absorbance just before the passage to the isotropic state. Such phenomenon can be interpreted by the discontinuous eharacter of the phase transition, both for the pure and doped LCs. The fluctuation phenomenon has been largely discussed in the literature and studied by many different experimental techniques and theoretical models such as the Landau theory [42] and using different experimental techniques [43-46]. 


\subsubsection{Differential scanning calorimetry}

The pure LCs, $5 \mathrm{CB}$ and $8 \mathrm{CB}$ have been extensively studied in the literature by different techniques, especially differential scanning calorimetry (DSC), in their pure state, doped by different compounds (dyes, nanoparticules,..) and dispersed in polymer matrices [47-49]. In this work, DSC analysis was applied for the same systems, to be compared to that of UVvisible spectroscopy. In addition, DSC measurements provide more information concerning the melting points and the enthalpies of the different phase transition. witch can be used for estimation of changes in the crystallinity of the LCs. All the results are calculated by Pyris Diamond DSC software.

The thermograms of L1-5CB and L1-8CB systems, resulting from the second heating cycle, are shown in figure 7 as examples. In DSC, the first heating cycle is used to erase the thermal history of the material, created as a result of the sample preparation. Before the first cycle, the material is not in its natural thermal state. The second cycle is then used because the sample has regained its basic thermal properties and hence the result is reproducible.

The method applied for determination of transition temperatures was from straight lines extended along the left-hand and right-hand parts of DSC tracks.

The nematic-isotropic transition temperatures of pure $5 \mathrm{CB}$ and $8 \mathrm{CB}$, were respectively 37,0 and $41,9^{\circ} \mathrm{C}$. The smectic-nematic transition of $8 \mathrm{CB}$ is observed at $34,7^{\circ} \mathrm{C}$. $\mathrm{L} 1-5 \mathrm{CB} 1,4 \mathrm{wt}-\%$ and $\mathrm{L} 2-5 \mathrm{CB} 0,7 \mathrm{wt}-\%$ show $\mathrm{T}_{\mathrm{NI}}$ values at 34,3 and $35,9^{\circ} \mathrm{C}$, respectively. As for $8 \mathrm{CB}$ systems, these transitions were observed towards 40,8 and $41,2^{\circ} \mathrm{C}$ for the higher concentration in L1 and L2. These values are of the same order of magnitude as those obtained by UV visible as well as these from literature [50]. Comparing these values with those given in table 1, as well as those from the literature [50], we can conclude that in general, DSC results are higher than those obtained from UV-vis technique. A difference of about 1,1 to $2^{\circ} \mathrm{C}$ for all systems can be explained by the difference in their heating rate employed in each case : $10^{\circ} / \mathrm{min}$ in DSC and 
steps of 0,1 and $0,5^{\circ} \mathrm{C}$ in $\mathrm{UV}$-vis. In this later, the sample seems to be permanently in a thermodynamic equilibrium, so the precision given by UV-vis measurement of transition temperatures is better than of DSC.

It should be noted that these systems show the quasi-identical same thermal behavior observed in both DSC and UV-visible spectroscopy, even if their results are slightly different. A regular decrease in transition temperatures as function of solute concentration was also observed, and proves to obey a linear law in such cases (Figure $6 \mathrm{~b}$ as example). even if the transition temperature values of pure $5 \mathrm{CB}$ and $8 \mathrm{CB}$ show in DSC an increase of 1.7 and $1.1^{\circ} \mathrm{C}$ respectively with regard to UV-visible spectroscopy. This can be related to the apparatts parameters used in each case.

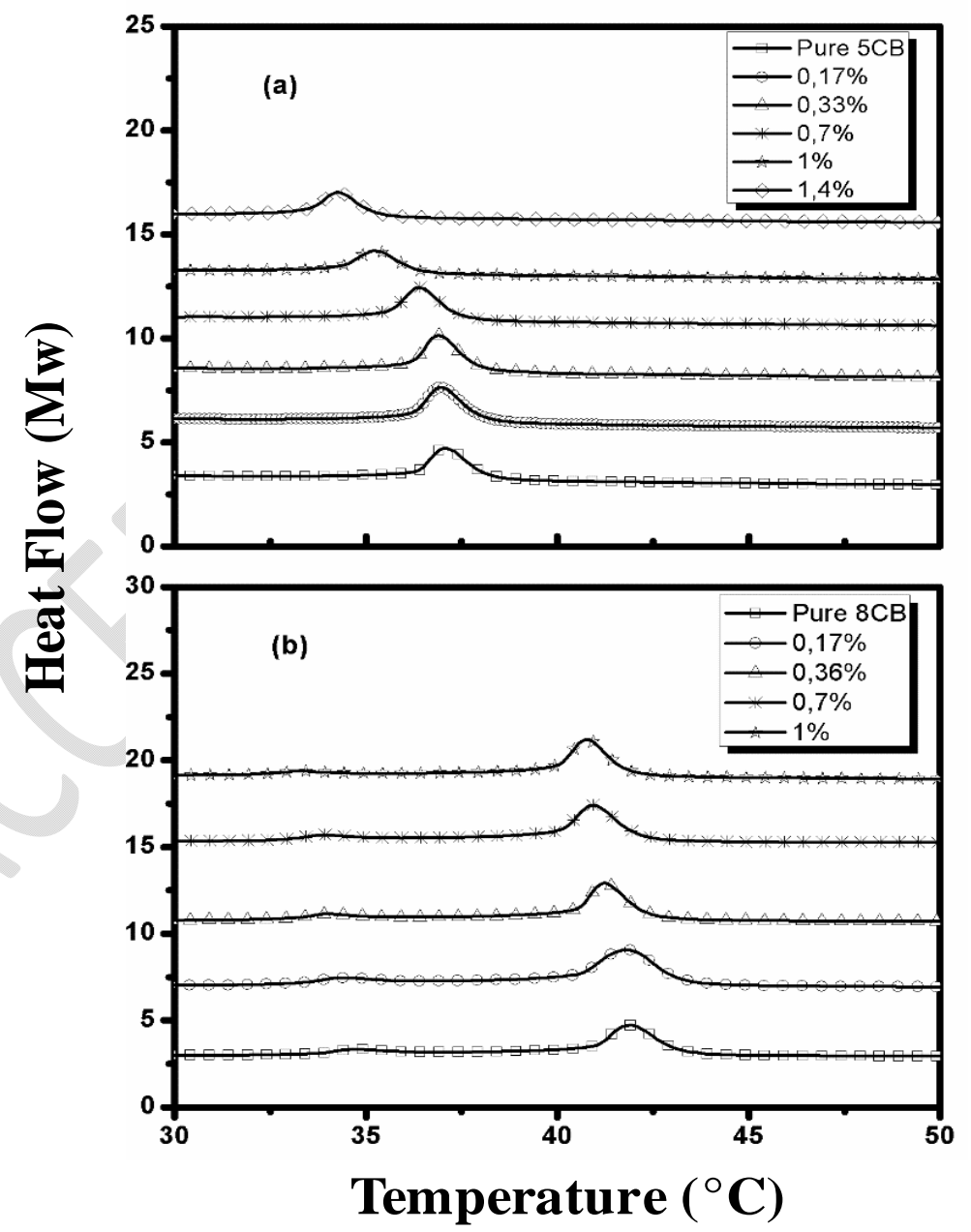

Figure 7 : Thermograms of (a) L1-5CB and (b) L1-8CB systems at different concentrations. 
The melting peaks of pure $5 \mathrm{CB}$ and $8 \mathrm{CB}$ undergo a displacement of about 0,12 to $0,62^{\circ} \mathrm{C}$, in the doped systems of $0,7 \mathrm{wt}-\%$. The enthalpies of the Nematic-Isotropic transitions of the same pure LCs are of 2,01 and 3,02 J/g respectively, reflecting the loose of their orientational order to became an isotropic systems, by releasing a small amount of energy. In the doped LCs, these values undergo a slight decrease in the doped systems which is of the order of about $0,2 \mathrm{~J} / \mathrm{g}$. Such a result shows that the orientational order erystallinity of the LCs is not strongly affected by the presence of these solutes, even at high concentration. The systems preserve, in general, the physical characteristics quasi-similar to those of the pure LCs, while acquiring new chemical and optical properties related to the structure of the solute inserted in the LC. This envisages their application in different fields of technology.

\subsubsection{Polarized Optical Microscopy}

The texture and the phase transition temperatures of LC-based systems were observed by polarized optical microscopy, using in the first cooling cycle, using a rate of $0.1^{\circ} \mathrm{C} / \mathrm{min}$. The pictures shown in figures 8 and 9 were taken, at different temperatures, around the phase transition of the LC-based systems. The Schlieren and/or focal conic textures, characterising the nematic and/or smectic phases of pure $5 \mathrm{CB}$ and $8 \mathrm{CB}$, are also observed in the doped LCs. This shows that the presence of the solute in the LCs don't destroy its characteristic texture nor its crystallinity, even at high concentration. In addition, no phase separation was observed on our scale, justifying the homogeneous dispersion of all solutes in the two LCs.

The nematic-isotropic transition of pure $5 \mathrm{CB}$ and $8 \mathrm{CB}$ are observed at 35.4 and $40.5^{\circ} \mathrm{C}$, respectively. These results are close to those obtained by UV-visible spectroscopy as well as those of the literature [51]. 

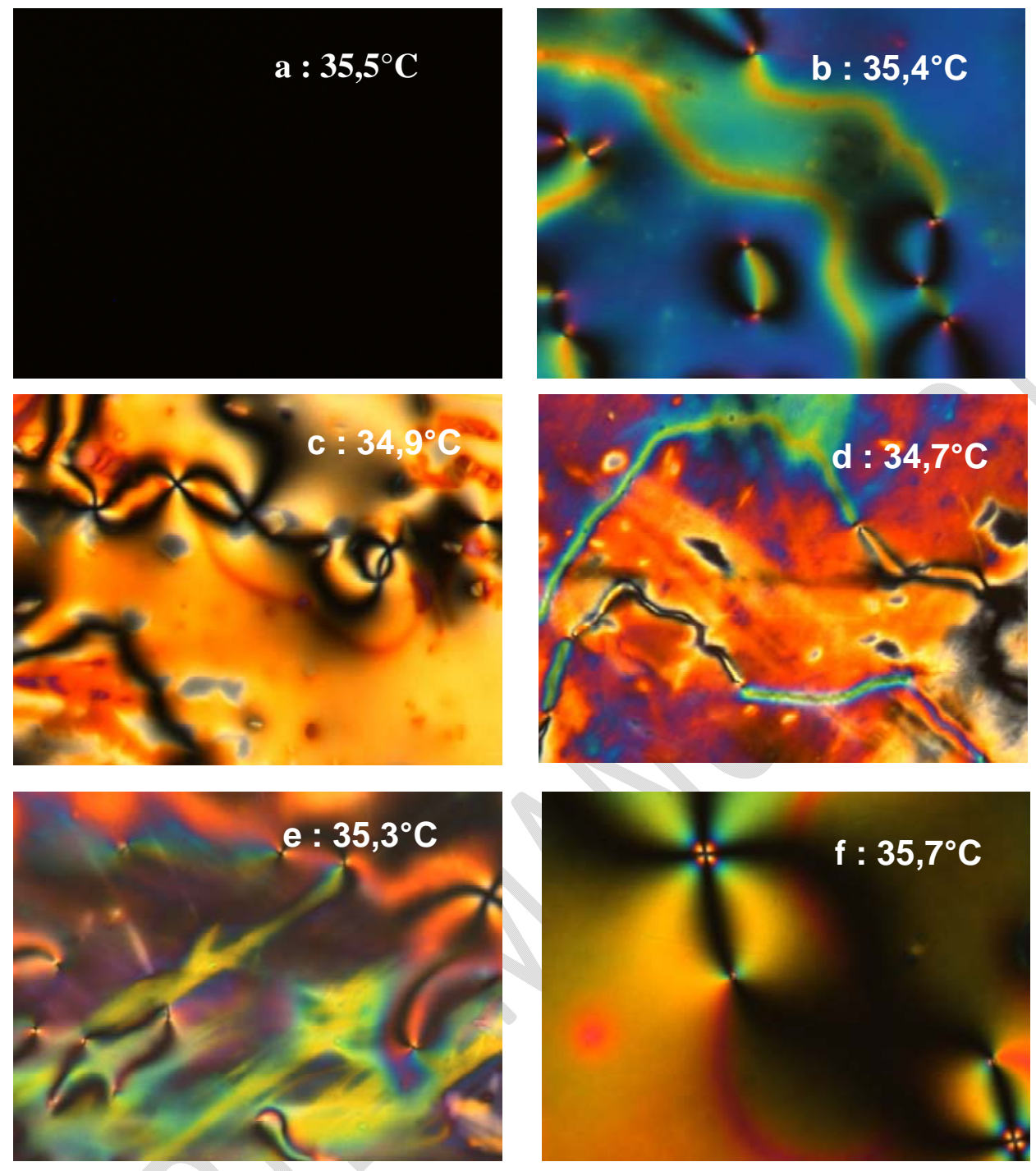

Figure 8 : Polarising optical microscopy pictures of 5CB based systems. Magnification x50. Images using crossed polarizers. Isotropic state of pure 5CB (a). Schlieren texture of the nematic phase taken around the transitional phase of pure 5CB (b), L1-5CB 0,7 wt-\% (c), L25CB 0,7 wt-\% (d), [L1Fe]-5CB 0,05 wt-\% (e) and [L2Ni]-5CB 0,05 wt-\% (f). 

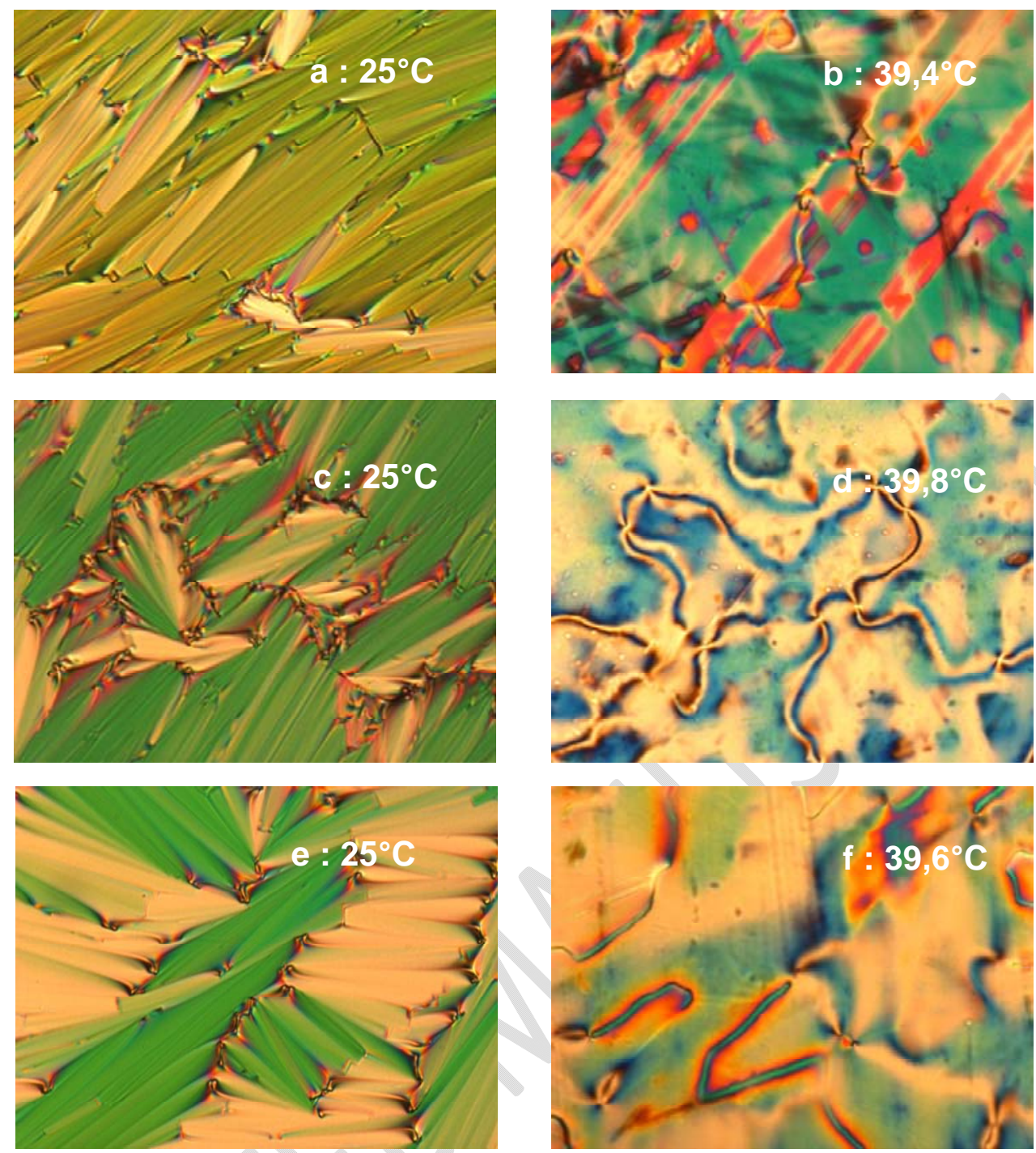

Figure 9 : Polarising optical microscopy pictures of $8 \mathrm{CB}$ based systems, taken at different phases. Magnification x50. Images using non-crossed polarizers. Conic focal texture of the smectic phase of pure 8CB (a), L1-8CB 0,36 wt-\% (c) and L2-8CB 0,70 wt-\% (e). Schlieren texture of the corresponding nematic phases of the same systems (b, $d$ and f respectively).

\section{Conclusion}

In this work, N,N'-bis(salicylidene)ethylenediamine (L1), N,N'-bis(5chlorosalicylidene)ethylene diamine (L2) and their metal complexes L1-Fe and L2-Ni were investigated and their structures elucidated by different analytical techniques. These systems 
were used as novel doping agents for two LCs of the cyanobiphenyl type, namely, 5CB and 8CB. The study of the mesophase of pure and doped LCs by these solutes was first carried out by UV-visible spectroscopy, equipped with a heating compartment. Such investigation is still rare in the literature, despite its simplicity and reliability.

The insertion of the elaborated systems in the LCs proves to be homogeneous, even at high concentration (up to $1.4 \mathrm{wt} \%$ ). This was justified by the linear evolution of their $\mathrm{T}_{\mathrm{NI}}$ and $\mathrm{T}_{\mathrm{SN}}$ as a function of the concentration in solute.

The peculiarity of the UV-visible technique consists in the possibility of observing the fluctuation phenomenon around the nematic-isotropic transition, in terms of the abrupt increase in absorbance and its drop to near zero, in a relatively low temperature range of about $\theta .1$ or $0.2^{\circ} \mathrm{C}$. Such a phenomenon is at the origin of the discontinuous character of the $\mathrm{N}-\mathrm{I}$ ins $L C$ singerat. On the other hand, the solute-solvent and solute-LC interactions were revealed by UV-visible spectra of the various solutes, carried out in ethanol, DMSO, acetonitrile, $5 \mathrm{CB}$ and $8 \mathrm{CB}$ at $42^{\circ} \mathrm{C}$.The mode of absorption in $5 \mathrm{CB}, 8 \mathrm{CB}$ and acetonitrile, having the same cyanide group,is found to be virtually identical. The $\mathrm{C} \equiv \mathrm{N}$ group seems to interact with the protons of the phenolic hydroxyls of the Schiff bases.

In addition, the results of the $\mathrm{T}_{\mathrm{NI}}$ and $\mathrm{T}_{\mathrm{SN}}$ obtained by UV-vis, DSC and POM for the doped LCs show that, in general, the nematic and/or smectic phases of these systems undergo a decrease of up to $2^{\circ} \mathrm{C}$ at maximum (for higher concentration). This makes it possible to assert that the LC character of these systems remains conserved. Phase separation phenomena were not detected in all cases considered here. Similarly, Schlieren and/or focal conic textures were clearly observed both in pure and doped LCs. The texture of these systems seems to be also conserved and further stabilized by the homogeneous insertion of the solute into the LC. 


\section{Acknowledgments}

This research project was financially supported by the Grant Ministry of Higher Education and Scientific Research (MESRS, Algeria), as well as the CNRS Department. The authors gratefully acknowledge the help provided by Dr. Christophe Beyens, from UMET of Lille1 University, DSC analysis.

\section{References :}

[1] Mucha M. Polymer as an important component of blends and composites with liquid crystals. Prog PolymSci. 2003; 28: 837-873.

[2] Doane JW, Vaz NA, Wu BG, et al. Field controlled light scattering from nematic microdroplets. Appl Phys Lett. 1986;48(4): 269-271.

[3] Grabchev I, Moneva I, Wolarz E, et al. Fluorescent 3-oxybenzanthrone dyes in liquid crystalline media. Dyes Pigm. 2003;58:1-6.

[4] Schmidt-Mende L, Fechtenkotter A, Mulle K, et al. SelfOrganized Discotic Liquid Crystals for High-Efficiency Organic Photovoltaics. Sci. 2001;293:1119-1122.

[5] Archambeau S, Bock H, Seguy I, et al. Organic solar cells with an ultra thin organized hole transport layer. J Mater Sci Mater Electron. 2007;18:919- 923.

[6] Heilmeier GH, Goldmacher JE. A new electric field controlled reflective optical storage effect in mixed liquid crystal systems. Proc IEEE. 1969;57:34-38.

[7] Matharu AS, Jeevaa S, Ramanujam PS. Liquid crystals for holographic optical data storage. ChemSoc Rev. 2007;36:1868-1880.

[8] Cretu C, Cseh L, Tang BJ, et al. Mononuclear Cu(II) complexes of novel salicylidene Schiff bases: synthesis and mesogenic properties. LiqCryst. 2015;42(8):1139-1147.

[9] Kuo HM, Ko WP, Hsu YT, et al. Mesogenic heterocycles derived from quinoxaline Schiff Bases. Tetrahedron. 2016;72:6321-6333. 
[10] Bagheri M, Pourmoazzen Z. Synthesis and properties of new liquid crystalline polyurethanes containing mesogenic side chain. Reactive Funct Poly. 2008;68:507-518.

[11] Zakerhamidi MS, Moghadam M, Ghanadzadeh A. Anisotropic and isotropic solvent effects on the dipole moment and photophysical properties of rhodamine dyes. J Lumi. 2012;132:931-937.

[12] Zakerhamidi MS, Ghanadzadeh A, Moghadam M. Effect of anisotropic and isotropic solvent on the dipole moment of coumarin dyes. Spectrochim Acta Part A. 2011;78:961-966.

[13] Tomylko S, Yaroshchuk O, Kovalchuk O, et al. Dielectric and electro-optical properties of liquid crystals doped with diamond nanoparticles. MolCrystLiqCryst. 2011;541:35-43.

[14] Lisetski LN, Fedoryako AP, Samoilov AN, et al. Optical transmission of nematic liquid crystal $5 \mathrm{CB}$ doped by single-walled and multi-walled carbon nanotubes. Eur Phys J E. $2014 ; 37: 1-7$

[15] Vimal T, Singh DP, Gupta SK, et al. Thermal and optical study of semiconducting CNTs-doped nematic liquid crystalline material. Phase Transitions. 2015;89:1-11.

[16] Basu R, Iannacchione GS. Carbon nanotube dispersed liquid crystal: A nano electromechanical system. Appl Phys Lett. 2008; 93:1-3.

[17] Ghanadzadeh Gilani A, Yazdanbakhsh MR, Mahmoodi N, et al. Solvatochromism and dichroism of fluorinated azoquinolin-8-ol dyes in liquid and liquid crystalline solutions. J Mol Liq. 2008;139:72-79.

[18] Ghanadzadeh A, Zeini A, Kashef A, et al. Solvent polarizability and anisotropy effects on the photophysical behavior of oxazine 1: An appropriatepolarizability indicator dye. Spectrochim Acta Part A. 2009;73:324-329.

[19] Khouba Z, Benabdallah T, Maschke U. Dispersion of pentadentate Schiff bases and their transition metal complexes in liquid crystals. MolCrystLiqCryst. 2009; 502:121-129. 
[20] Khouba Z, Benabdallah T, Maschke U. Spectrophotometric study of liquid crystals containing pentadentate Schiff base type systems. Phys Procedia. 2009;2:1305-1311.

[21] Makwana NG, Doshi AV. Study of binary systems and determination of latent transition temperatures (LTTs) of non mesogenic components. J IndChem Soc. 2008;85:262-266.

[22] HadjYoucef M, Benabdallah T, Ilikti H, et al. Equilibrium studies on the synergic liquidliquid extraction process of copper(II) from sulphate media with mixtures of some bidentate mono-Schiff bases and acyclic polyether non-ionic surfactant in chloroform. Solvent Extr Ion Exch. 2008;26:534-555.

[23] Al Zoubi W. Solvent extraction of metal ions by use of Schiff bases. J Coord Chem. 2013;66(13):2264-2289.

[24] Khouba Z, Benabdallah T, Maschke U. Spectrophotometric investigation of interaction between iodine and pentadentate Schiff base ligands. Spectrochim Acta A. 2014;125: 61-66.

[25] Ghosh S, Ghosh A. Coordination of metalloligand [NiL] (H2L = salen type N2O2 Schiff base ligand) to the f-block elements: Structural elucidation and spectrophotometric investigation. InorgChim Acta. 2016;442:64-69.

[26] Sivasubramanian VK, Ganesan M, Rajagopal S, et al. Iron(III)- Salen Complexes as Enzyme Models: Mechanistic Study of Oxo(salen)iron Complexes Oxygenation of Organic Sulfides. J Org Chem. 2002; 67:1506-1514.

[27] Cozzi PG. Metal-Salen Schiff base complexes in catalysis: practical aspects. ChemSoc Rev. 2004; 33:410-421.

[28] Canali L, Sherrington DC. Utilisation of homogeneous and supported chiral metal(salen) complexes in asymmetric catalysis. ChemSoc Rev. 1999;28:85-93.

[29] Negm NA, Zaki MF. Corrosion inhibition efficiency of nonionic Schiff base amphiphiles of p-aminobenzoic acid for aluminum in 4N HCL. Colloids Surf A: PhysicochemEng Aspects. 2008;322:97-102. 
[30] Agrawal YK, Talati JD, Shah MD, et al. Schiff bases of ethylenediamine as corrosion inhibitors of zinc in sulphuric acid. Corros Sci. 2004;46:633-651.

[31] Taha ZA, Ajlouni AM, Al-Hassan KA, et al. Syntheses, characterization, biological activity and fluorescence properties of bis-(salicylaldehyde)-1,3-propylenediimine Schiff base ligand and its lanthanide complexes. Spectrochim Acta Part A.2011;81:317-323.

[32] Golcu A, Tumer M, Demirelli H, et al. Cd(II) and Cu(II) complexes of polydentate Schiff base ligands: Synthesis, characterization, properties and biological activity. Inorganica Chim. Acta. 2005;358:1785-1797.

[33] Amer S, El-Wakiel N, El-Ghamry H. Synthesis, spectral, antitumor and antimicrobial studies on $\mathrm{Cu}(\mathrm{II})$ complexes of purine and triazole Schiff base derivatives. J Mol Struct. 2013;1049:326-335.

[34] Pahontu E, Ilies DC, Shova S, et al. Synthesis, Characterization, Crystal Structure and Antimicrobial Activity of Copper(II) Complexes with the Schiff Base Derived from 2Hydroxy-4-Methoxybenzaldehyde. Mol. 2015;20:5771-5792.

[35] Zolezzi S, Decinti A, Spodine E. Syntheses and characterization of copper (II) complexes with Schiff-base ligands derived from ethylenediamine, diphenylethylenediamine and nitro, bromo and methoxy salicylaldehyde. Polyhedron. 1999;18:897-904.

[36] Aranha PE, Dos Santos MP, Romera S, et al. Synthesis, characterization, and spectroscopic studies of tetradentate Schiff base chromium(III) complexes. Polyhedron. 2007;26:1373-1382.

[37] Hriharan M, Urbach FL. The Stereochemistry of Tetradentate Schiff Base Complexes of Cobalt(II). Inorg Chem. 1969;8:556-559.

[38] Rubavathi DSR, Balakuma S. Iron(III)-Salen-Catalyzed H2O2 Oxidation of Dibenzyl Sulphide. Asian J. Chem. 2010 ; 22 (6) : 4891-4897.

[39] SivasubramanianVK, Ganesan M, Rajagopal S, et al. Iron(III)-Salen complexes as en 
zyme models: mechanistic study of Oxo(salen)iron complexes oxygenation of organic sulfides. J Org Chem. 2002 ; 67(5) : 1506-1514.

[40] Uzarevic K, Rubcic M, Stilinovic V, et al. Keto-enol tautomerism inasymmetric Schiff bases derived from p-phenylenediamine, J Mol Struct. 2010;984:232-239.

[41] Grabchev I, Moneva I, Bojinov V, et al. Synthesis and properties of fluorescent 1,8naphthalimide dyes for application in liquid crystal displays. J Mater Chem. 2000;10:12911296.

[42] Stinson TW, Litster JD. Pretransitional phenomena in the isotopic phase of a nematic liquid crystal. Phys Rev Lett. 1970;25(8):503-506.

[43] Zink H, DeJeu WH. A light-Scattering Study of pretransitional behavior around the isotropic-nematic phase transition in alkylcyanobiphenyls. Mol. Cryst. Liq. Cryst. 1985 ; $124: 287-304$

[44] De Schrijver P, Van Dael W, Thoen J. Surface-induced pretransitional order in the isotropic phase near the isotropic-nematic phase transition. Liqcryst. 1996; 21(5): 745-749. [45]Nastishin YA, Liu H, Shiyanovskii SV, et al. Pretransitional fluctuations in the isotropic phase of a lyotropic chromonic liquid crystal. Phys Rev E. 2004; 70 (051706): 1-8. [46] Van Roie B, Leys J, Denolf K, et al. Weakly first-order character of the nematic-isotropic phase transition in liquid crystals. Phys Rev E. 2005 ; 72(041702) : 1-8.

[47] Benmouna F, Daoudi A, Roussel F, et al. Effect of Molecular Weight on the Phase Diagram and Thermal Properties of Poly(styrene)/8CB Mixtures. Macromolecules. 2000;33:960-967.

[48] Perju E, Paslaru E, Marin L. Polymer-dispersed liquid crystal composites for bioapplications: thermotropic, surface and optical properties. LiqCryst. 2015;42(3):370-382.

[49] Park S, Kim HK, Hong JW. Investigation of the photopolymerization-induced phase separation process in polymer dispersed liquid crystal. Polym Test. 2010;29:886-893. 
[50] Sharma D, MacDonald JC, Iannacchione GS. Thermodynamics of activated phase transitions of 8CB: DSC and MC calorimetry. J Phys Chem B. 2006;110:16679-16684.

[51] Marin L, Popescu MC, Zabulica A, et al. Chitosan as matrix for bio-polymer dispersed liquid crystal systems. CarbohydrPolym. 2013;95:16-24. 